\title{
Transesophageal Echocardiography Guided Novel Technique for the Negotiation of Dual Stage Cavo-atrial Cannula into Inferior Vena Cava in a Patient with Large Eustachian Valve: A Case Report
}

\author{
${ }^{1}$ Krishna Prasad Gourav, ${ }^{2}$ Azeez Aspari, ${ }^{3}$ Sunder Lal Negi, ${ }^{4}$ Srinath Damodaran, ${ }^{5}$ Shyam Thingnam
}

\begin{abstract}
Eustachian valve is an embryonic remnant of the right valve of sinus venosus. In adults it may be completely absent or may appear as a thin flap. However, in some cases it may persist as a long mobile structure which can be mistaken as patent foramen ovale or cor-triatrium dexter. Sometimes it can obstruct the single stage cavo-atrial cannula while inserting into inferior vena cava (IVC). We hereby discuss a technique which helped in the successful cannulation of the dual stage cavo-atrial cannula into the IVC in a patient with a giant eustachian valve with the help of transesophageal echocardiography (TEE).
\end{abstract}

Keywords: Eustachian valve, Dual stage Cavo-atrial cannula, Transesophageal echocardiography

How to cite this article: Gourav KP, Aspari A, Negi SL, Damodaran S, Thingnam S. Transesophageal Echocardiography Guided Novel Technique for the Negotiation of Dual Stage Cavo-atrial Cannula into Inferior Vena Cava in a Patient with Large Eustachian Valve: A Case Report. J Perioper Echocardiogr 2018;6(2):57-59.

Source of support: Nil

Conflict of interest: None

\section{INTRODUCTION}

Eustachian valve is an embryonic remnant of the right valve of the sinus venosus. In adults, this structure may be completely absent or it may appear as a thin flap. ${ }^{1-2}$ However, in some patients, it may persist as a mobile long structure in the right atrium. Although benign in nature, big eustachian valves are associated with some rare

1,2,4Senior Resident, ${ }^{3}$ Assistant Professor, ${ }^{5}$ Professor and Head

1,2Department of Anesthesia, Postgraduate Institute of Medical Education and Research, Chandigarh, India

${ }^{3}$ Department of Anesthesia and Intensive Care, Postgraduate Institute of Medical Education and Research, Chandigarh, India

${ }^{4}$ Department of Cardiac Anesthesiology, Postgraduate Institute of Medical Education and Research, Chandigarh, India

${ }^{5}$ Department of CTVS, Postgraduate Institute of Medical Education and Research, Chandigarh, India

Corresponding Author: Sunder Lal Negi, Assistant Professor, Department of Anesthesia and Intensive Care, Postgraduate Institute of Medical Education and Research, Chandigarh, India e-mail: dr.sundernegi@gmail.com complications. TEE provides an important diagnostic and functional information during the perioperative period in cardiac surgery. It also helps in guiding the surgeon during venous cannulation for its proper placement. We hereby report a case showing the importance of TEE, which guided us in the negotiation of dual stage cavoatrial cannulation in a patient with the large eustachian valve.

\section{CASE DESCRIPTION}

A 58-year-old female patient came to our institute with a complaint of chest pain on mild exertion. An electrocardiogram revealed ST segment depression in V3, V4 and V5 leads. A normal biventricular function with mild mitral regurgitation was reported on transthoracic echocardiography (TTE). The coronary angiogram revealed $60 \%$ stenosis of the left main coronary artery (CA), $90 \%$ stenosis of the left anterior descending artery, $80 \%$ stenosis of the right coronary artery and $80 \%$ stenosis of the left circumflex artery. Hence, the patient was scheduled for coronary artery bypass grafting (CABG) surgery after a thorough pre-anesthetic checkup. On the day of surgery in the operating room, a $16 \mathrm{G}$ intravenous (IV) cannula was placed in the right arm and a 20G cannula was placed in the left radial artery. Anesthesia was induced with $200 \mathrm{mcg}$ IV fentanyl, $60 \mathrm{mg}$ IV Propofol and $6 \mathrm{mg}$ IV vecuronium. The trachea was intubated with a $7.5 \mathrm{~mm}$ cuffed endotracheal tube after achieving adequate muscle relaxation. An adult TEE probe (6VT-D GE healthcare, Vivid E9 Norway) was inserted into the esophagus and the preoperative TTE findings were confirmed. A large 23mm eustachian valve was seen in the mid-esophageal bicaval view. Initially, it was misinterpreted as a part of inter-atrial septum and a misdiagnosis of patent foramen ovale was made (Fig. 1, Video 1). However, after careful examination in multiple views, it was diagnosed as a eustachian valve. After midline sternotomy and adequate anticoagulation with heparin, aortic cannulation was performed successfully. While introducing dual stage cavo-atrial venous cannulae a resistance was felt by the surgeon at 


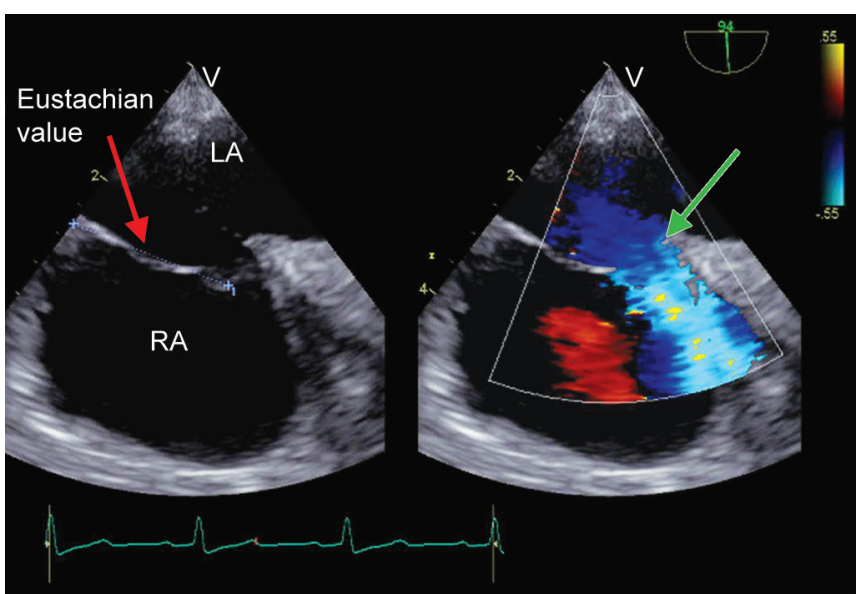

Fig. 1: A mid-esophageal bicaval view showing a gaint $2.3 \mathrm{~cm}$ eustachian valve. It was misinterpreted as a part of interatrial septum and misdiagnosis of patent foramen ovale (green arrow) was made. LA, left atrium; RA, right atrium.

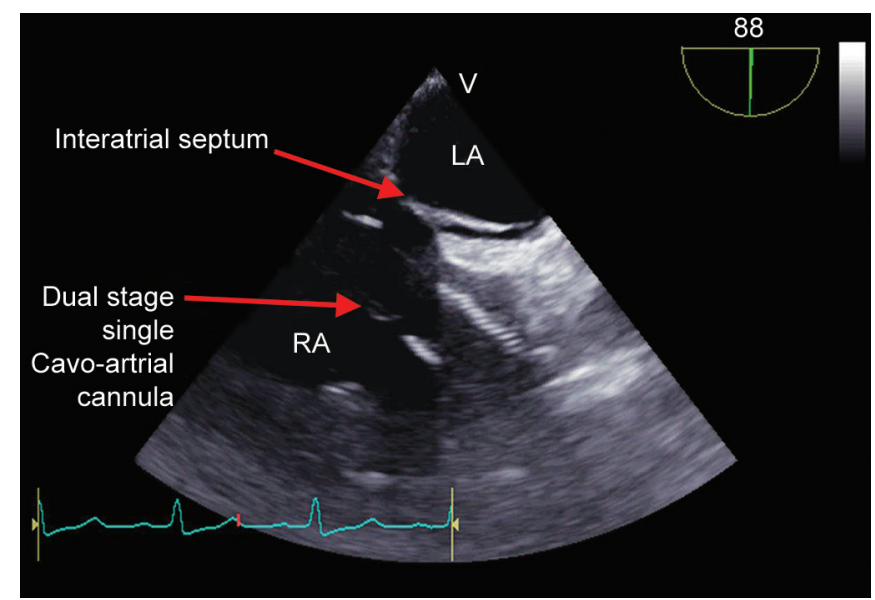

Fig. 3: Mid-esophageal bicaval view showing a dual stage cavoatrial cannula directed towards interatrial septum and then advanced into the inferior vena cava. LA, left atrium; RA, right atrium.

the lower aspect of the right atrium with inability to pass the cannula from the right atrium into the inferior vena cava. TEE exam in mid-esophageal bicaval view revealed the eustachian valve as the cause of the obstruction of the venous cannula (Fig. 2, Video 2). Hence, surgeon was suggested to direct the venous cannula towards the inter-atrial septum and then direct towards the inferior vena cava (IVC). The dual stage venous cannula was successfully cannulated into the IVC through this maneuver under the guidance of TEE (Fig. 3, Video 3). Following which, cardiopulmonary bypass (CPB) was then successfully initiated with adequate venous return. After CABG, the $\mathrm{CPB}$ was terminated with the support of noradrenaline $0.03 \mathrm{mcg} / \mathrm{kg} / \mathrm{min}$ and the patient was shifted to the cardiac intensive care unit with stable hemodynamics.

\section{DISCUSSION}

The eustachian valve is a venous valve located at the junction of IVC and right atrium. It helps in directing

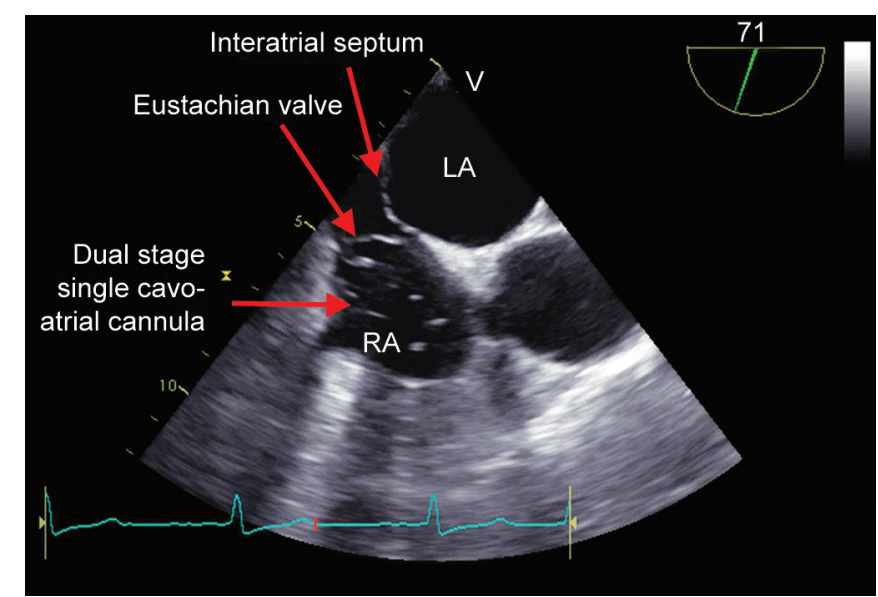

Fig. 2: Mid-esophageal modified bicaval view at 710 rotation showing a eustachian valve obstructing the insertion of dual stage cavoatrial cannula into inferior vena cava. LA, left atrium; $\mathrm{RA}$, right atrium.

oxygen-rich blood from the IVC towards the foramen ovale in fetal life. In adults, it is non-functional and is considered as a benign rudimentary structure. It is visualized in only $0.2 \%$ of adults undergoing routine echocardiographic examinations. ${ }^{3}$ The persistent eustachian valve has a large spectrum of variability in size, thickness, shape, and texture. At one end of the spectrum, it disappears totally or is represented only by a thin ridge. At the other extreme, it persists as a mobile, elongated structure projecting several centimeters into the right atrial cavity. When it is quite large, it can be mistaken for patent foramen ovale or cor-triatrium dexter. $^{4-5}$ In this index case, the eustachian valve was initially misinterpreted as a part of inter-atrial septum and diagnosed to have a patent foramen ovale. However, on comprehensive TEE examination in multiple views, it was diagnosed as a eustachian valve.

The presence of a large eustachian valve is usually a benign echo finding. However, in some situations, it may be clinically significant. Complications associated with a large eustachian valve includes infective endocarditis, thrombosis and possibly subsequent pulmonary embolism. ${ }^{6,7}$ Occasionally, a big eustachian valve can produce obstruction to flow entering from the IVC to the right atrium. Sometimes, eustachian valve can impair venous drainage by obstructing venous cannula during cardiopulmonary bypass. ${ }^{8}$ Eckhard et al. reported a case of a giant eustachian valve obstructing the advancement of a dual stage venous cannula into the IVC even after several attempts under the guidance of TEE. ${ }^{9}$ Hence, they used a big single stage venous cannula and kept in the right atrium. In the index case, dual stage venous cannula was successfully advanced into the IVC on 2nd attempt when the surgeons, tirected the venous cannula towards the inter-atrial septum followed by advancement into the IVC under the guidance of TEE in mid-esophageal bicaval 
view. To the best of our knowledge, this is the first case reporting a successful cannulation of IVC with dual stage venous cannula in a patient with a large eustachian valve. In addition to eustachian valve, other benign structures like thebesian valve and Chiari network are also prone to obstruct cannulation of cardiac structures. ${ }^{9-11}$

\section{CONCLUSION}

Large eustachian valve can obstruct the placement of dual stage venous cannula while instituting cardiopulmonary bypass. Anesthesiologist can help the surgeon in guiding the dual stage venous cannula past a large eustachian valve with the help of intraop TEE. Redirecting the dual stage venous cannula towards the inter-atrial septum and then advancing into IVC may be one such maneuver to help avoiding the cannula getting obstructed by eustachean valve.

\section{REFERENCES}

1. Grande AM, Fiore A, Massetti M, Viganò M. Iatrogenic circumflex coronary lesion in mitral valve surgery: Case report and review of the literature. Tex Heart Inst J 2008;35:179-183.

2. Ucar O, Canbay A, Diker E, Aydogdu S. Long Eustachian valve interfering with the access to coronary sinus during biventricular pacemaker implantation. AnadoluKardiyolDerg. 2010;10(2):185-186.
3. Schuchlenz HW, Saurer G, Weihs W, Rehak P. Persisting eustachian valve in adults: Relation to patent foramen ovale and cerebrovascular events. J Am SocEchocardiogr2004;17:231-233.

4. Yavuz T, Nazli C, Kinay O, Kutsal A. Giant eustachian valve with echocardiographic appearance of divided right atrium. Tex Heart Inst J 2002;29:336-338.

5. Becker A, Buss M, Sebening W, Meisner H, Döhlemann C. Acute inferior cardiac inflow obstruction resulting from inadvertent surgical closure of a prominent Eustachian valve mistaken for an atrial septal defect. PediatrCardiol1999;20:155-157.

6. Barriales V, Tamargo JA, Aguado MG, Martin M, Rondan J, Segovia E, Morís C. Floating thrombi on the Eustachian valve as a complication of venous thromboembolic disease. Int J Cardiol2004;93:289-291.

7. Wong RC, Teo SG, Yeo TC. An unusual right-sided endocarditis: a case report of eustachian valve endocarditis. Int J Cardiol2006;109:406-407.

8. Iannoli ED. The use of transesophageal echocardiography for differential diagnosis of poor venous return during cardiopulmonary bypass. AnesthAnalg. 2007 Jul;105(1):43-44.

9. Schmid E, Scheule A, Locke A, et al: Echocardiographicguided placement of venous cannula due to inferior vena cava obstruction through a large eustachian valve. AnesthAnalg 111:76-78, 2010

10. Kuroda M, Takahashi T, Mita N, et al: Difficult cannulation of the coronary sinus due to a large Thebesian valve. AnesthAnalg 116:563-566, 2013.

11. Teo EY, Ittleman F, Hamlin MP: A Chiari network and difficult cannulation of the coronary sinus for retrograde perfusion. Anesth Analg 111:79-81, 2010 